\title{
NONSMOOTH DUALITY, SANDWICH, AND SQUEEZE THEOREMS
}

\author{
A. S. LEWIS ${ }^{\dagger}$ AND R. E. LUCCHETTI ${ }^{\ddagger}$
}

\begin{abstract}
Given a nonlinear function $h$ separating a convex and a concave function, we provide various conditions under which there exists an affine separating function whose graph is somewhere almost parallel to the graph of $h$. Such results blend Fenchel duality with a variational principle and are closely related to the Clarke-Ledyaev mean value inequality.
\end{abstract}

Key words. sandwich theorem, squeeze theorem, Fenchel duality, variational principle, mean value inequality, Clarke subdifferential

AMS subject classifications. 49J52, 90C46, 26D07

PII. S0363012998334213

1. Introduction. The central theorems in this paper blend two completely distinct types of result, both fundamental in optimization theory: Fenchel duality and variational principles. The simplest version of Fenchel duality states that for any convex functions $f$ and $g$ on $\mathbf{R}^{n}$ satisfying $f \geq-g$, a regularity condition implies the set

$$
L \stackrel{\text { def }}{=}\left\{y \in \mathbf{R}^{n}: f^{*}(y)+g^{*}(-y) \leq 0\right\}
$$

is nonempty (where $f^{*}$ is the Fenchel conjugate of $f$ ). Geometrically, this means there exists an affine function sandwiched between $f$ and $-g$. On the other hand, one of the easiest examples of a variational principle states that if $h$ is a locally Lipschitz function bounded below on $\mathbf{R}^{n}$, then $h$ has arbitrarily small Clarke subgradients:

$$
0 \in \operatorname{cl}(\operatorname{Im} \partial h) .
$$

Geometrically, there are points where the graph of $h$ is almost horizontal (in a certain nonsmooth sense).

The theorems we discuss here combine the features of both results above. We consider functions $f, g$, and $h$ as before, now satisfying $f \geq h \geq-g$, and under various regularity conditions we prove

$$
L \cap \operatorname{cl}(\operatorname{Im} \partial h) \neq \emptyset .
$$

Geometrically, there are affine functions between $f$ and $-g$ whose graphs are somewhere almost parallel to the graph of $h$.

As we show by means of various examples, the existence of a suitable affine separating function depends on both local and asymptotic properties of the three functions. Hence the regularity conditions we need to impose combine assumptions on the domains of the primal functions, $f$ and $g$, and of their conjugates, $f^{*}$ and $g^{*}$, as well as local and global growth conditions on $h$.

\footnotetext{
*Received by the editors February 18, 1998; accepted for publication (in revised form) May 27, 1999; published electronically February 9, 2000. This research was partially supported by the Natural Sciences and Engineering Research Council of Canada.

http://www.siam.org/journals/sicon/38-2/33421.html

${ }^{\dagger}$ Department of Combinatorics and Optimization, University of Waterloo, Waterloo, Ontario, Canada N2L 3G1 (aslewis@orion.uwaterloo.ca; http://orion.uwaterloo.ca/ aslewis).

${ }^{\ddagger}$ Politecnico di Milano, Facoltá di Ingegneria di Como, P.le Gerbetto 6, 22100 Como, Italy (rel@komodo.ing.unico.it).
} 
The key tool for our results is a recent, somewhat surprising mean value inequality of Clarke-Ledyaev [3], rephrased as a hybrid sandwich theorem in [6]. We illustrate the application of this type of result with two apparently simple but rather remarkable consequences. First, any convex function $f$ and locally Lipschitz function $h \leq f$ satisfy

$$
\operatorname{dom} f^{*} \cap \operatorname{cl}(\operatorname{Im} \partial h) \neq \emptyset
$$

Second (a "squeeze theorem"), any locally Lipschitz functions $p \geq h \geq q$ with $p(0)=$ $h(0)=q(0)$ satisfy

$$
\partial p(0) \cap \partial h(0) \cap \partial q(0) \neq \emptyset .
$$

We have not been able to find simple proofs or references for either of these two results. ${ }^{1}$

With the exception of this last squeeze theorem, our results do not appear to be substantially easier with the assumption that $h$ is smooth (in which case $\partial h$ reduces to the singleton $\nabla h$ ). We believe they provide further evidence of the depth, applicability, and fundamental nature of the Clarke-Ledyaev inequality in optimization theory.

2. Notation and preliminary results. We begin by reviewing some basic ideas from convex analysis (see [7]). Given a convex set $A \subset \mathbf{R}^{n}$, we denote by aff $A$ the smallest affine space containing $A$ and by ri $A$ the set of the internal points of $A \subset$ aff $A$ (with the induced topology). Observe that ri $A$ is a nonempty convex set. Given a function $f: \mathbf{R}^{n} \rightarrow[-\infty, \infty]$, we denote its effective domain by

$$
\operatorname{dom} f \stackrel{\text { def }}{=}\left\{x \in \mathbf{R}^{n}: f(x)<\infty\right\}
$$

and by epi $f$ its epigraph, the set

$$
\text { epi } f \stackrel{\text { def }}{=}\left\{(x, r) \in \mathbf{R}^{n} \times \mathbf{R}: r \geq f(x)\right\},
$$

a convex set if and only if $f$ is convex. The hypograph of the function $-g$ is instead

$$
\operatorname{hyp}(-g) \stackrel{\text { def }}{=}\left\{(x, r) \in \mathbf{R}^{n} \times \mathbf{R}: r \leq-g(x)\right\},
$$

again a convex set if and only if $g$ is convex. The epigraph of $f$ is closed if and only if $f$ is lower semicontinuous in the usual sense.

We shall write $f \in \Gamma_{0}$ to mean that epi $f$ is nonempty, closed, and convex and does not contain vertical lines.

For a set $A$, let $I_{A}$ be the indicator function of the set $A$,

$$
I_{A}(x)= \begin{cases}0 & \text { if } x \in A \\ \infty & \text { otherwise }\end{cases}
$$

In particular, $I_{k B}$ denotes the indicator function of the ball centered at $0 \in \mathbf{R}^{n}$ and with radius $k$.

\footnotetext{
${ }^{1}$ Subsequent investigations revealed alternative approaches to the last theorem independent of the Clarke-Ledyaev result [1]. Nonetheless, the original approach we present here remains attractive for its transparency.
} 
The Fenchel conjugate of a function $f: \mathbf{R}^{n} \rightarrow[-\infty, \infty]$ is the function $f^{*}: \mathbf{R}^{n} \rightarrow$ $[-\infty, \infty]$ defined by

$$
f^{*}(y) \stackrel{\text { def }}{=} \sup _{x \in \mathbf{R}^{n}}\{\langle y, x\rangle-f(x)\}
$$

a convex lower semicontinuous function (even if $f$ is not) which belongs to $\Gamma_{0}$ if $f$ does. Furthermore, $f=f^{* *}$, providing $f \in \Gamma_{0}$.

The function $f$ is said to be cofinite if its conjugate $f^{*}$ satisfies $\operatorname{dom} f^{*}=\mathbf{R}^{n}$. It is easy to see this is equivalent to saying that $\lim _{\|x\| \rightarrow \infty} \frac{f(x)}{\|x\|}=\infty[5$, Chapter 10, Proposition 1.3.8].

The subdifferential of a convex function $f$ at a point $x \in \operatorname{dom} f$ is the closed convex set

$$
\partial f(x) \stackrel{\text { def }}{=}\left\{y \in \mathbf{R}^{n}: f(z) \geq f(x)+\langle y, z-x\rangle \text { for all } z \in \mathbf{R}^{n}\right\} .
$$

The fundamental connection between the subdifferentials of a function and of its Fenchel conjugate is shown by the following Fenchel identity:

$$
y \in \partial f(x) \Longleftrightarrow f(x)+f^{*}(y)=\langle y, x\rangle .
$$

It follows, in particular, that $y \in \partial f(x)$ if and only if $x \in \partial f^{*}(y)$, providing $f \in \Gamma_{0}$.

Given two functions $p, q: \mathbf{R}^{n} \rightarrow[-\infty, \infty]$, we define the infimal convolution between them by

$$
(p \square q)(x)=\inf _{y \in \mathbf{R}^{n}}\{p(y)+q(x-y)\},
$$

a convex function if $p$ and $q$ are, possibly assuming the value $-\infty$, and that may fail to be lower semicontinuous. Finally, for a function $p \in \Gamma_{0}$, we denote by

$$
p_{k} \stackrel{\text { def }}{=} p \square k\|\cdot\|,
$$

the infimal convolution between $p$ and $k\|\cdot\|:$ this function is the largest $k$-Lipschitz function minorizing $p$. For more about convex functions, the interested reader is invited to consult [4], [5], [7].

Next we briefly consider the subdifferential of a locally Lipschitz function $h$ : $U \rightarrow \mathbf{R}$, where $U$ is an open subset of $\mathbf{R}^{n}$. This notion is not uniquely defined in the literature, and here we make the choice of the Clarke subdifferential (see [2]) which is more suited for our scopes, as an example in the final section will show. To define it, first let us introduce the notion of generalized directional derivative of $h$ at the point $x$ in the direction $v$ :

$$
h^{\circ}(x, v) \stackrel{\text { def }}{=} \limsup _{\substack{z \rightarrow x \\ t \searrow 0}} \frac{h(z+t v)-h(z)}{t} .
$$

The function $v \mapsto h^{\circ}(x, v)$ is everywhere finite, subadditive, and positively homogeneous; hence, in particular, it is continuous and convex. Then the subdifferential of $h$ at $x$ is defined as

$$
\partial h(x) \stackrel{\text { def }}{=}\left\{y \in \mathbf{R}^{n}:\langle y, v\rangle \leq h^{\circ}(x, v) \text { for all } v \in \mathbf{R}^{n}\right\},
$$

a nonempty closed convex set. Moreover, if $k$ is a Lipschitz constant for $h$, the subdifferential is norm-bounded by $k$. In particular, the multifunction $\partial h$ is bounded on 
bounded sets. Observe that the Clarke subdifferential of $h$ at $x$ is the same set as the (convex) subdifferential of the function $v \mapsto h^{\circ}(x, v)$ at $v=0$, a simple but useful property we shall use throughout this paper.

For more about nonsmooth analysis for locally Lipschitz functions, the interested reader is invited to consult [2].

In this paper we shall deal with two convex functions $f, g \in \Gamma_{0}$ and a locally Lipschitz function $h$ such that $f \geq h \geq-g$. For a moment, let us focus on the problem of nonemptyness of the set

$$
L \stackrel{\text { def }}{=}\left\{y \in \mathbf{R}^{n}: f^{*}(y)+g^{*}(-y) \leq 0\right\} .
$$

This set can be characterized in a more geometric way, as the following easy proposition states.

Proposition 2.1. Let $f, g \in \Gamma_{0}$. Then, for $y \in \mathbf{R}^{n}$,

$$
f^{*}(y)+g^{*}(-y) \leq 0
$$

if and only if there exists $a \in \mathbf{R}$ such that

$$
f(x) \geq a+\langle y, x\rangle \geq-g(x) \text { for all } x \in \mathbf{R}^{n} .
$$

Thus the problem of nonemptyness of $L$ is equivalent to finding an affine separator lying below $f$ and above $g$. This can be stated in terms of a separation problem for the sets epi $f$ and hyp $(-g)$. The assumption $f \geq-g$ ensures that

$$
\text { ri epif } \cap \text { ri hyp }(-g)=\emptyset \text {, }
$$

(see [4, Chapter 4, Proposition 1.1.9]) and this in turn implies that epi $f$ and hyp $(-g)$ can be separated by a hyperplane [4, Chapter 3, Theorem 4.1.4]. However, it can happen that the only separating hyperplane is vertical, which unfortunately says nothing about nonemptyness of $L$.

The first result stating that $L$ is nonempty is the following well-known Fenchel duality theorem [7, Theorem 31.1], which in our setting can be rephrased in the following way.

THEOREM 2.1. Let $f, g \in \Gamma_{0}$ be such that $f \geq-g$ and suppose

$$
\operatorname{ri}(\operatorname{dom} f) \cap \operatorname{ri}(\operatorname{dom} g) \neq \emptyset .
$$

Then there exists $y \in \mathbf{R}^{n}$ such that

$$
f^{*}(y)+g^{*}(-y) \leq 0 .
$$

We illustrate the role of the assumption on the domains of $f$ and $g$ with the help of the following four examples, where the set $L$ is always empty.

EXAMPLE 2.1.

$$
\begin{aligned}
& f(x)= \begin{cases}-\sqrt{x} & \text { if } x \geq 0, \\
\infty & \text { otherwise },\end{cases} \\
& g(x)= \begin{cases}0 & \text { if } x=0, \\
\infty & \text { otherwise }\end{cases}
\end{aligned}
$$

Here ri $(\operatorname{dom} f) \cap \operatorname{ri}(\operatorname{dom} g)=\emptyset$. 
EXAMPLE 2.2.

$$
\begin{aligned}
& f(u, v)= \begin{cases}-1 & \text { if } u v \geq 1, u \geq 0 \\
\infty & \text { otherwise }\end{cases} \\
& g(u, v)= \begin{cases}0 & \text { if } u \geq 0, v=0, \\
\infty & \text { otherwise. }\end{cases}
\end{aligned}
$$

Here we have $\operatorname{dom} f \cap \operatorname{dom} g=\emptyset$.

EXAMPLE 2.3.

$$
\begin{aligned}
& f(u, v)=\left\{\begin{array}{cc}
u & \text { if } v=-1, \\
\infty & \text { otherwise }
\end{array}\right. \\
& g(u, v)=\left\{\begin{array}{lc}
0 & \text { if } v=0, \\
\infty & \text { otherwise }
\end{array}\right.
\end{aligned}
$$

Here the distance between $\operatorname{dom} f$ and $\operatorname{dom} g$ is 1 .

In the last two examples the domains of $f$ and $g$ do not intersect, while in the first example a crucial role is played by the fact that $\inf (f+g)=0$. In the following example $\inf (f+g)>0$, and yet there is no affine separator. Observe that such an example could not be provided in one dimension [4, Chapter 1, Remark 3.3.4].

EXAMPLE 2.4.

$$
\begin{gathered}
f(u, v)= \begin{cases}1-2 \sqrt{u v} & \text { if } u, v \geq 0, \\
\infty & \text { otherwise, }\end{cases} \\
g(u, v)= \begin{cases}1-2 \sqrt{-u v} & \text { if } u \leq 0, v \geq 0, \\
\infty & \text { otherwise. }\end{cases}
\end{gathered}
$$

A straightforward calculation shows

$$
\begin{aligned}
& f^{*}\left(u^{*}, v^{*}\right)=\left\{\begin{array}{cc}
-1 & \text { if } u^{*} \leq 0, u^{*} v^{*} \geq 1, \\
\infty & \text { otherwise, }
\end{array}\right. \\
& g^{*}\left(u^{*}, v^{*}\right)=\left\{\begin{array}{cc}
-1 & \text { if } u^{*} \geq 0, u^{*} v^{*} \leq-1, \\
\infty & \text { otherwise. }
\end{array}\right.
\end{aligned}
$$

Thus the set $L$ is empty.

3. Sandwich theorems. We turn now to the case of three functions $f, g$, and $h$, such that $f$ and $g$ are convex, $h$ is locally or globally Lipschitz, and $f \geq h \geq-g$. Examples 2.2, 2.3, and 2.4 show that the existence of a locally Lipschitz function $h$ between $f$ and $-g$ (take $h(x, y)=-x y$ in all cases) does not change the situation: there is no affine separator.

First let us recall now some known results.

TheOREM 3.1 (see [6, Theorem 2]). Let $C$ be a nonempty convex compact set in $\mathbf{R}^{n}$. Let $f, g \in \Gamma_{0}$ and with domains contained in $C$. Let $h: \mathbf{R}^{n} \rightarrow \mathbf{R}$ be Lipschitz on a neighborhood of $C$. Suppose moreover $f \geq h \geq-g$ on $C$. 
Then there exist $c \in C$ and $y \in \partial h(c)$ such that

$$
f^{*}(y)+g^{*}(-y) \leq 0 .
$$

Boundedness of $C$ can be relaxed at the expense of requiring more about the functions $f$ and $g$ and/or about the function $h$. Specifically, we have the following two results.

TheOREm 3.2 (see [6, Theorem 7]). Let $C$ be a nonempty closed convex set in $\mathbf{R}^{n}$. Let $f, g \in \Gamma_{0}$ be cofinite, with domains contained in $C$. Moreover suppose

$$
\operatorname{int}(\operatorname{dom} f) \cap \operatorname{int}(\operatorname{dom} g) \neq \emptyset .
$$

Let $h: \mathbf{R}^{n} \rightarrow \mathbf{R}$ be locally Lipschitz on a neighborhood of $C$ and suppose $f \geq h \geq-g$ on $C$.

Then there exist $c \in C$ and $y \in \partial h(c)$ such that

$$
f^{*}(y)+g^{*}(-y) \leq 0 .
$$

Theorem 3.3 (see [6, Theorem 8]). Let $C$ be a nonempty closed convex set in $\mathbf{R}^{n}$. Let $f, g \in \Gamma_{0}$ be cofinite, with domains contained in $C$. Let $h: \mathbf{R}^{n} \rightarrow \mathbf{R}$ be globally Lipschitz on a neighborhood of $C$ and suppose $f \geq h \geq-g$ on $C$.

Then there exist $c \in C$ and $y \in \partial h(c)$ such that

$$
f^{*}(y)+g^{*}(-y) \leq 0 .
$$

Observe one does not need a qualification condition on the domains of $f$ and $g$ if $h$ is globally Lipschitz. In the last two theorems, however, cofiniteness is required, which can be regarded as a (strong) qualification condition on the domains of the conjugates.

The first result we want to prove deals simply with the existence of the affine separator. To prove it, we need the following proposition about regularizing Fenchel problems.

Proposition 3.1. Suppose $p, q \in \Gamma_{0}$ and

$$
(\bullet) \quad \operatorname{ri}(\operatorname{dom} p) \cap \operatorname{ri}(\operatorname{dom} q) \neq \emptyset \text {. }
$$

Then, for all large $k$, we have

$$
\inf (p+q)=\inf \left(p_{k}+q_{k}\right)
$$

and

$$
\operatorname{argmin}(p+q)=\operatorname{argmin}\left(p_{k}+q_{k}\right)
$$

Proof. To prove the first equality, we need to prove only $\inf (p+q) \leq \inf \left(p_{k}+q_{k}\right)$. There is nothing to prove if $\inf (p+q)=-\infty$. Therefore, let us assume it is finite. (It cannot be $\infty$ because of $(\bullet)$.) By Fenchel duality, there is $y \in \mathbf{R}^{n}$ such that

$$
-\inf (p+q)=p^{*}(y)+q^{*}(-y) .
$$

Take $k>\|y\|$. Then 


$$
\begin{gathered}
-\inf (p+q)=p^{*}(y)+q^{*}(-y)=\left(p^{*}+I_{k B}\right)(y)+\left(q^{*}+I_{k B}\right)(-y) \\
=\left(p_{k}\right)^{*}(y)+\left(q_{k}\right)^{*}(-y) \geq \inf _{z \in \mathbf{R}^{n}}\left(\left(p_{k}\right)^{*}(z)+\left(q_{k}\right)^{*}(-z)\right) \\
=-\inf \left(p_{k}+q_{k}\right) \geq-\inf (p+q) .
\end{gathered}
$$

This shows the first equality and also that $y$ as above is optimal for the problem of minimizing, on $\mathbf{R}^{n},\left(p_{k}\right)^{*}(\cdot)+\left(q_{k}\right)^{*}(-\cdot)$.

Now, writing down optimality conditions, we obtain, using $k>\|y\|$,

$$
\begin{aligned}
x \in \operatorname{argmin}(p+q) & \Leftrightarrow p(x)+q(x)=-p^{*}(y)-q^{*}(-y), \\
& \Leftrightarrow x \in \partial p^{*}(y) \cap \partial q^{*}(-y), \\
& \Leftrightarrow x \in \partial\left(p^{*}+I_{k B}\right)(y) \cap \partial\left(q^{*}+I_{k B}\right)(-y), \\
& \Leftrightarrow x \in \partial\left(p_{k}\right)^{*}(y) \cap \partial\left(q_{k}\right)^{*}(-y), \\
& \Leftrightarrow x \in \operatorname{argmin}\left(p_{k}+q_{k}\right) .
\end{aligned}
$$

We begin our sequence of main results by proving some variants of Fenchel duality, where the usual regularity condition is replaced by the existence of a Lipschitz separator.

Theorem 3.4. For $f, g \in \Gamma_{0}$, suppose

$$
\operatorname{ri}\left(\operatorname{dom} f^{*}\right) \cap \operatorname{ri}\left(-\operatorname{dom} g^{*}\right) \neq \emptyset .
$$

Suppose further there exists a locally Lipschitz function $h$ such that $f \geq h \geq-g$. Then there is $y \in \mathbf{R}^{n}$ such that

$$
f^{*}(y)+g^{*}(-y) \leq 0 .
$$

(Moreover, if $\inf (f+g)=0$, then such a $y$ can be found in the range of $\partial h$.)

Proof. From Proposition 3.1, applied to $p=f^{*}$ and $q(\cdot)=g^{*}(-\cdot)$, we have

$$
\inf \left(\left(f^{*}\right)_{k}(\cdot)+\left(g^{*}\right)_{k}(-\cdot)\right)=\inf \left(f^{*}(\cdot)+g^{*}(-\cdot)\right)
$$

and

$$
\operatorname{argmin}\left(\left(f^{*}\right)_{k}(\cdot)+\left(g^{*}\right)_{k}(-\cdot)\right)=\operatorname{argmin}\left(f^{*}(\cdot)+g^{*}(-\cdot)\right)
$$

for all large $k$. Apply Theorem 3.1 to the functions $f+I_{k B}, h$, and $g+I_{k B}$, for large $k$, and the set $C=k B$, to find $y_{k} \in \operatorname{Im} \partial h$ such that

$$
\left(f^{*}\right)_{k}\left(y_{k}\right)+\left(g^{*}\right)_{k}\left(-y_{k}\right) \leq 0 .
$$

If

$$
y_{k} \in \operatorname{argmin}\left(\left(f^{*}\right)_{k}(\cdot)+\left(g^{*}\right)_{k}(-\cdot)\right)
$$

(as in the case when $\inf (f+g)=0)$, then we deduce $f^{*}\left(y_{k}\right)+g^{*}\left(-y_{k}\right) \leq 0$ and we conclude. Otherwise, for all large $k$,

$$
0>\inf \left(\left(f^{*}\right)_{k}(\cdot)+\left(g^{*}\right)_{k}(-\cdot)\right)=\inf \left(f^{*}(\cdot)+g^{*}(-\cdot)\right) .
$$


Thus there is $y \in \mathbf{R}^{n}$ such that $f^{*}(y)+g^{*}(-y) \leq 0$, as required.

We provided here the result when $\inf (f+g)=0$ for the sake of completeness. However observe that under the assumptions of Theorem $3.4 \inf f+g$ is attained. In this circumstance the squeeze theorem in the next section will provide a more precise result.

With respect to the role of the assumptions in Theorem 3.4, Example 2.1 shows the set $L$ can be empty if we do not assume the existence of a locally Lipschitz function sandwiched between $f$ and $-g$, while Example 2.2 shows the necessity of the qualification condition

$$
\operatorname{ri}\left(\operatorname{dom} f^{*}\right) \cap \operatorname{ri}\left(-\operatorname{dom} g^{*}\right) \neq \emptyset .
$$

We turn now to the problem of providing conditions under which the slope of an affine separator can be found in the closure of the range of the Clarke subdifferential of the separating function $h$. To do this, we prove first the following proposition.

Proposition 3.2. Suppose $f, g \in \Gamma_{0}$ satisfy $f \geq-g$. For $k=1,2, \ldots$, define

$$
L_{k} \stackrel{\text { def }}{=}\left\{y \in \mathbf{R}^{n}:\left(f^{*}\right)_{k}(y)+\left(g^{*}\right)_{k}(-y) \leq 0\right\}
$$

and

$$
L \stackrel{\text { def }}{=}\left\{y \in \mathbf{R}^{n}: f^{*}(y)+g^{*}(-y) \leq 0\right\} .
$$

Then $L_{k}$ is a decreasing collection of closed convex sets containing $L$, and

$$
y_{k} \in L_{k}, \quad y_{k} \rightarrow y \quad \text { implies } \quad y \in L .
$$

If moreover the condition

$$
0 \in \operatorname{int}(\operatorname{dom} f-\operatorname{dom} g)
$$

holds, then the sets $L_{k}$ for large $k$ are all contained in a compact set.

Proof. Since $\left(f^{*}\right)_{k} \leq\left(f^{*}\right)_{k+1} \leq f^{*}$, clearly $L \subset L_{k+1} \subset L_{k}$, for all $k>0$. Let us prove that, if $y_{k}$ is such that $y_{k} \rightarrow y$ and

$$
\left(f^{*}\right)_{k}\left(y_{k}\right)+\left(g^{*}\right)_{k}\left(-y_{k}\right) \leq 0 \text { for all } k,
$$

then

$$
f^{*}(y)+g^{*}(-y) \leq 0 .
$$

From Proposition 2.1 there exists $a_{k} \in \mathbf{R}$ such that

$$
f(x) \geq a_{k}+\left\langle y_{k}, x\right\rangle \geq-g(x) \text { for all } x \in k B .
$$

It is easy to show the sequence $\left\{a_{k}\right\}$ is bounded, so it has some cluster $a \in \mathbf{R}$. (Use the boundedness of $\left\{y_{k}\right\}$ and the existence of an element $x \in \operatorname{dom} f \cap \operatorname{dom} g$.) It follows that

$$
f(x) \geq a+\langle y, x\rangle \geq-g(x) \text { for all } x \in \mathbf{R}^{n},
$$

so $y \in L$. We have proved the first part of the claim. Now define a function

$$
v(w)=\inf _{x \in \mathbf{R}^{n}}(f(x+w)+g(x))
$$


and a sequence of functions decreasing pointwise to $v$,

$$
v^{k}(w)=\inf _{x \in \mathbf{R}^{n}}\left(\left(f+I_{k B}\right)(x+w)+\left(g+I_{k B}\right)(x)\right) .
$$

Observe that $\left(v^{k}\right)^{*}(y)=\left(f^{*}\right)_{k}(y)+\left(g^{*}\right)_{k}(-y)$ and $v^{*}(y)=f^{*}(y)+g^{*}(-y)$ and that $\operatorname{dom} v=\operatorname{dom} f-\operatorname{dom} g$, so that $0 \in \operatorname{int}(\operatorname{dom} v)$.

Since $v$ is continuous at 0 , there exist reals $r>0$ and $\alpha$ and a cube $C$ such that $r B \subset C \subset \operatorname{int}(\operatorname{dom} v)$ and $v \leq \alpha-1$ on $C$. Hence, for large $k$ we have $v^{k} \leq \alpha$ on each vertex of $C$ and hence on $r B$, so $\left(v^{k}\right)^{*}(w) \geq r\|w\|-\alpha$ for all points $w$ in $\mathbf{R}^{n}$, and therefore $L_{k} \subset(\alpha / r) B$.

We are now ready for a new result.

TheOREM 3.5. For $f, g \in \Gamma_{0}$, and locally Lipschitz $h: \mathbf{R}^{n} \rightarrow \mathbf{R}$ satisfying $f \geq h \geq-g$, suppose

$$
0 \in \operatorname{int}(\operatorname{dom} f-\operatorname{dom} g) .
$$

Then

$$
\exists y \in \operatorname{cl}(\operatorname{Im} \partial h): f^{*}(y)+g^{*}(-y) \leq 0 .
$$

Proof. Apply Theorem 3.1 to the functions $f+I_{k B} \geq h \geq-\left(g+I_{k B}\right)$, for large $k$. Then there exists

$$
y_{k} \in \operatorname{Im}(\partial h):\left(f^{*}\right)_{k}\left(y_{k}\right)+\left(g^{*}\right)_{k}\left(-y_{k}\right) \leq 0 .
$$

By Proposition 3.2 the sequence $\left(y_{k}\right)$ clusters and any cluster point satisfies the required property.

We intend now to prove that the constraint qualification in Theorem 3.5 can be replaced by an assumption involving the growth of $f$ and $h$ at infinity. To do this, we need the following proposition.

Proposition 3.3. For $f \in \Gamma_{0}$ and locally Lipschitz $h$ satisfying $f \geq h$, suppose

$$
\liminf _{\|x\| \rightarrow \infty} \frac{f(x)}{\|x\|}>\max \left\{\limsup _{\|x\| \rightarrow \infty} \frac{h(x)}{\|x\|}, 0\right\} .
$$

Then, for all large $k, f_{k} \geq h$.

Proof. Let $0<a<b$ and $c$ be such that

$$
\frac{f(x)}{\|x\|} \geq b, \quad \frac{h(x)}{\|x\|} \leq a,
$$

for all $x$ such that $\|x\| \geq c$. Then there exists $r b f \in \mathbf{R}$ such that

$$
f(x) \geq r+b\|x\| \text { for all } x \in \mathbf{R}^{n},
$$

and $f$ has bounded level sets. For the sake of contradiction, suppose there exists, for each $k \in \mathbf{N}, x_{k}$ such that $f_{k}\left(x_{k}\right)<h\left(x_{k}\right)$. Two cases can occur.

(i) $\left(x_{k}\right)$ is unbounded. Taking a subsequence, we can suppose $\left\|x_{k}\right\| \rightarrow \infty$. For $k>b$, we have $f_{k}\left(x_{k}\right) \geq r+b\left\|x_{k}\right\|$. It follows that

$$
a\left\|x_{k}\right\| \geq h\left(x_{k}\right)>f_{k}\left(x_{k}\right) \geq r+b\left\|x_{k}\right\|,
$$


a contradiction.

(ii) $\left(x_{k}\right)$ is bounded. Again taking a subsequence, we can suppose $x_{k} \rightarrow x$. Pick $m>\|x\|$ and $r$ so that $h$ is $r$-Lipschitz on $m B$. Since $f$ has compact level sets, for each $k$ there is $y_{k}$ such that $h\left(x_{k}\right)>f_{k}\left(x_{k}\right)=f\left(y_{k}\right)+k\left\|x_{k}-y_{k}\right\|$. As $h\left(x_{k}\right) \rightarrow h(x)$, for large $k$ one has

$$
f\left(y_{k}\right) \leq f\left(y_{k}\right)+k\left\|x_{k}-y_{k}\right\| \leq h(x)+1 .
$$

Thus $\left(y_{k}\right)$ is bounded and, taking another subsequence, we can suppose $y_{k} \rightarrow y$. Since

$$
k\left\|x_{k}-y_{k}\right\| \leq h(x)+1-\inf f \text { for all } k,
$$

we deduce $y=x$. Thus, for large $k, x_{k}, y_{k} \in m B$, so

$$
h\left(x_{k}\right) \leq h\left(y_{k}\right)+r\left\|x_{k}-y_{k}\right\| \leq f\left(y_{k}\right)+k\left\|x_{k}-y_{k}\right\|<h\left(x_{k}\right),
$$

a contradiction.

THEOREM 3.6. For $f, g \in \Gamma_{0}$ and locally Lipschitz $h: \mathbf{R}^{n} \rightarrow \mathbf{R}$ satisfying $f \geq h \geq-g$, suppose

$$
\liminf _{x \rightarrow \infty} \frac{f(x)}{\|x\|}>\max \left\{\limsup _{x \rightarrow \infty} \frac{h(x)}{\|x\|}, 0\right\} .
$$

Then

$$
\exists y \in \operatorname{cl}(\operatorname{Im} \partial h): f^{*}(y)+g^{*}(-y) \leq 0 .
$$

Proof. From Proposition 3.3, $f_{k} \geq h \geq-g$ for large $k$. Since we know $\operatorname{dom} f_{k}-$ $\operatorname{dom} g=\mathbf{R}^{n}$, Theorem 3.5 implies there exists $y \in \mathrm{cl} \operatorname{Im} \partial h$ with

$$
f^{*}(y)+g^{*}(-y) \leq\left(f_{k}\right)^{*}(y)+g^{*}(-y) \leq 0 .
$$

The proof of the theorem above relies on the fact that we are able to construct a function $p \in \Gamma_{0}$ such that $f \geq p \geq h$ and whose domain contains internal points. However, to do this is not always possible, as the following example shows.

EXAMPLE 3.1. Let

$$
f(u, v)=\left\{\begin{array}{lr}
0 & \text { if } v=0 \\
\infty & \text { otherwise }
\end{array}\right.
$$

and $h(u, v)=|u v|$. Suppose the convex function $p$ satisfies $f \geq p \geq h$. Then clearly $p(u, 0)=0$ for all $u$. For any real $u$ and $v$ and positive integer $r$,

$$
\begin{aligned}
\frac{1}{r} p(u, v) & =\frac{1}{r} p(u, v)+\frac{r-1}{r} p(u, 0) \\
& \geq p\left(u, \frac{v}{r}\right) \\
& =p\left(u, \frac{v}{r}\right)+p(r, 0) \\
& \geq 2 p\left(\frac{u+r}{2}, \frac{v}{2 r}\right) \\
& \geq\left|\frac{(u+r) v}{2 r}\right| \\
& \rightarrow \frac{|v|}{2}
\end{aligned}
$$


as $r \rightarrow \infty$. Hence $p(u, v)=+\infty$ whenever $v \neq 0$, so $p=f$.

The next result deals with the case of $h$ being globally Lipschitz.

THEOREM 3.7. For $f, g \in \Gamma_{0}$ and globally Lipschitz $h: \mathbf{R}^{n} \rightarrow \mathbf{R}$, suppose $f \geq h \geq-g$. Then

$$
\exists y \in \operatorname{cl}(\operatorname{Im} \partial h): f^{*}(y)+g^{*}(-y) \leq 0
$$

Proof. Apply Theorem 3.3 to the functions $f+I_{k B}, h$, and $g+I_{k B}$ to obtain the existence of $y_{k} \in \operatorname{Im} \partial h$ such that

$$
\left(f+I_{k B}\right)^{*}\left(y_{k}\right)+\left(g+I_{k B}\right)^{*}\left(-y_{k}\right) \leq 0 .
$$

Since $h$ is globally Lipschitz, $\left(y_{k}\right)$ has a cluster point $y$. Then we conclude with the help of Proposition 3.2.

The next example shows that in general no $y \in \operatorname{Im} \partial h$ satisfies $f^{*}(y)+g^{*}(-y) \leq 0$.

EXAMPLE 3.2. Let $f(x)=|x|$,

$$
\begin{gathered}
g(x)=\left\{\begin{array}{lr}
1+x^{2} & \text { if } x \geq 0, \\
\infty & \text { otherwise, }
\end{array}\right. \\
h(x)=\left\{\begin{array}{lr}
x-\exp (-x) & \text { if } x \geq 0, \\
2 x-1 & \text { otherwise. }
\end{array}\right.
\end{gathered}
$$

Then all the assumptions of Theorem 3.5 are fulfilled, and moreover $h$ is globally Lipschitz.

We end the section by proving a unilateral result which can be regarded as a generalized variational principle.

ThEOREM 3.8. For $f \in \Gamma_{0}$ and locally Lipschitz $h: \mathbf{R}^{n} \rightarrow \mathbf{R}$, suppose $f \geq h$. Then

$$
\operatorname{cl}(\operatorname{Im} \partial h) \cap \operatorname{dom} f^{*} \neq \emptyset
$$

Proof. Choose any point $z \in \operatorname{dom} f$ and real $k>\|z\|$. Define $g(\cdot)=I_{k B}(\cdot)-$ $\inf _{k B} h$ and apply Theorem 3.5.

The special case $f=0$ gives the well-known variational result that a locally Lipschitz function $h$ which is bounded above satisfies $0 \in \operatorname{cl}(\operatorname{Im} \partial h)$.

4. Squeeze theorems. In this section we specialize the situation studied before. We shall make the further assumption that there is a point where the three functions are equal. In this case, as we shall see, we are able to provide more precise results.

We shall start with the following easy proposition, that we state without proof.

Proposition 4.1. For $f, g \in \Gamma_{0}$ satisfying $f \geq-g$, suppose there exists $x$ such that $f(x)=-g(x)$. Then

$$
\left\{y: f^{*}(y)+g^{*}(-y) \leq 0\right\}=\partial f(x) \cap-\partial g(x) .
$$

We prove now a "convex" squeeze theorem.

THEOREM 4.1. For $f, g \in \Gamma_{0}$ and locally Lipschitz $h: \mathbf{R}^{n} \rightarrow \mathbf{R}$ satisfying $f \geq h \geq-g$, suppose there exists $x \in \mathbf{R}^{n}$ such that $f(x)=-g(x)$. Then

$$
\partial f(x) \cap \partial h(x) \cap-\partial g(x) \neq \emptyset .
$$


Proof. Without loss of generality, we can suppose $x=0$. For each positive integer $r$, as $f \geq h \geq-g$ on $\frac{1}{r} B$, we can apply Theorem 3.1 to find $x_{r} \in \frac{1}{r} B$ and $y_{r} \in \partial h\left(x_{r}\right)$ with

$$
\left(f+I_{\frac{1}{r} B}\right)^{*}\left(y_{r}\right)+\left(g+I_{\frac{1}{r} B}\right)^{*}\left(-y_{r}\right) \leq 0 .
$$

By Proposition 4.1

$$
y_{r} \in \partial\left(f+I_{\frac{1}{r} B}\right)(0) \cap-\partial\left(g+I_{\frac{1}{r} B}\right)(0)=\partial f(0) \cap-\partial g(0) .
$$

Since $\partial h$ is locally bounded, there exists a subsequence $\left(y_{r_{k}}\right)$ of $\left(y_{r}\right)$ converging to some $y$, and since $x_{r_{k}} \rightarrow 0$ and $\partial h$ is closed, $y \in \partial h(0)$.

The next squeeze theorem deals instead with three locally Lipschitz functions. To prove it, we need the following proposition.

Proposition 4.2. Let $f: \mathbf{R}^{n} \rightarrow \mathbf{R}$ be locally Lipschitz and suppose $\delta>0$. Then

$$
f(0)+f^{\circ}(0, x)+\delta\|x\|>f(x)
$$

for all small nonzero $x$.

Proof. Suppose that $f(0)=0$, that $f$ is $k$-Lipschitz near 0 , and that, for the sake of contradiction, there is a sequence $\left(x_{r}\right)$ such that $x_{r} \neq 0$ for all $r$ and $x_{r} \rightarrow 0$, with

$$
f^{\circ}\left(0, x_{r}\right)+\delta\left\|x_{r}\right\| \leq f\left(x_{r}\right) .
$$

Thus

$$
f^{\circ}\left(0, \frac{x_{r}}{\left\|x_{r}\right\|}\right)+\delta \leq \frac{f\left(x_{r}\right)}{\left\|x_{r}\right\|} .
$$

Suppose, without loss of generality, $\frac{x_{r}}{\left\|x_{r}\right\|} \rightarrow d$. Then

$$
\begin{aligned}
\limsup _{r \rightarrow \infty} f^{\circ}\left(0, \frac{x_{r}}{\left\|x_{r}\right\|}\right)+\delta & \leq \limsup _{r \rightarrow \infty} \frac{f\left(x_{r}\right)}{\left\|x_{r}\right\|} \\
& =\limsup _{r \rightarrow \infty} \frac{f\left(\left\|x_{r}\right\| d\right)+f\left(x_{r}\right)-f\left(\left\|x_{r}\right\| d\right)}{\left\|x_{r}\right\|} \\
& \leq \limsup _{r \rightarrow \infty} \frac{\left.f\left(\left\|x_{r}\right\| d\right)+k\left\|x_{r}-\right\| x_{r}\|d\|\right)}{\left\|x_{r}\right\|} \\
& \leq f^{\circ}(0, d) .
\end{aligned}
$$

It follows that

$$
f^{\circ}(0, d)+\delta \leq f^{\circ}(0, d)
$$

which is impossible.

TheOrEm 4.2. Suppose $f, h, g: \mathbf{R}^{n} \rightarrow \mathbf{R}$ are three locally Lipschitz functions such that $f \geq h \geq g$. Moreover, suppose $f(x)=g(x)$ for some $x$. Then

$$
\partial f(x) \cap \partial h(x) \cap \partial g(x) \neq \emptyset .
$$

Proof. Suppose, without loss of generality, $f(0)=h(0)=g(0)=0$. By Proposition 4.2 , for each $r \in \mathbf{N}$, there exists $\varepsilon_{r}>0$ such that

$$
f^{\circ}(0, x)+\frac{\|x\|}{r} \geq h(x) \geq-\left((-g)^{\circ}(0, x)+\frac{\|x\|}{r}\right)
$$


for all $x$ such that $\|x\| \leq \varepsilon_{r}$. Now, take $\varepsilon<\varepsilon_{r}$. Then

$$
f^{\circ}(0, x)+\frac{\|x\|}{r}+I_{\varepsilon B}(x) \geq h(x) \geq-\left((-g)^{\circ}(0, x)+\frac{\|x\|}{r}+I_{\varepsilon B}(x)\right)
$$

for all $x \in \mathbf{R}^{n}$. We can then apply Theorem 4.1 to get an element $y_{r}$ such that

$$
\begin{aligned}
y_{r} & \in \partial\left(f^{\circ}(0, \cdot)+\frac{\|\cdot\|}{r}+I_{\varepsilon B}(\cdot)\right)(0) \cap \partial h(0) \\
& \cap-\partial\left((-g)^{\circ}(0, \cdot)+\frac{\|\cdot\|}{r}+I_{\varepsilon B}(\cdot)\right)(0) \\
= & \left(\partial f(0)+\frac{1}{r} B\right) \cap \partial h(0) \cap\left(\partial g(0)+\frac{1}{r} B\right) .
\end{aligned}
$$

Since $y_{r} \in \partial h(0)$, the sequence $\left(y_{r}\right)$ is bounded and any of its cluster points does the job.

Corollary 4.1. Let $f_{1} \geq f_{2} \geq \cdots f_{k}: \mathbf{R}^{n} \rightarrow \mathbf{R}$ be locally Lipschitz. Suppose $f_{1}(0)=\cdots=f_{k}(0)$. Then

$$
\bigcap_{i=1}^{k} \partial f_{i}(0) \neq \emptyset,
$$

provided at least one of the following conditions holds:

- $k=1,2,3$;

- at least one $f_{i}$ is smooth;

- $n=1,2$.

Proof. The cases $k=2$ and the case when $f_{i}$ is smooth follow from the sum rule applied to $f_{1}-f_{2}$ and $f_{j}-f_{i}$, respectively. The case $k=3$ is Theorem 4.2 and the cases $n=1,2$ are consequences of Theorem 4.2 and Helly's theorem.

5. Final remarks. We have seen some sandwich and squeeze theorems, dealing with convex and locally Lipschitz functions. While the convex subdifferential is standard, there are several notions of subdifferential for locally Lipschitz functions. Here we use the Clarke subdifferential rather than, for instance, the approximate subdifferential, because the latter is not suitable for the results we seek. Consider the following simple example.

EXAMPLE 5.1. Let

$$
\begin{gathered}
f(x)=I_{[-1,1]}(x), \\
g(x)=|x|+I_{[-1,1]}(x),
\end{gathered}
$$

and

$$
h(x)=-|x| .
$$

Then $f \geq h \geq-g$ and $h$ is (globally) Lipschitz. However

$$
L=\left\{y: f^{*}(y)+g^{*}(y) \leq 0\right\}=\{0\},
$$

while the approximate subdifferential of $h$ is the set $\{-1,1\}$.

Finally, here is a list of questions we leave to the interested reader. 
Question 1. Does

$$
\operatorname{ri}(\operatorname{dom} f) \cap \operatorname{ri}(\operatorname{dom} g) \neq \emptyset
$$

imply

$$
L \cap \operatorname{cl}(\operatorname{Im} \partial h) \neq \emptyset ?
$$

Question 2. Does

$$
\operatorname{ri}\left(\operatorname{dom} f^{*}\right) \cap \operatorname{ri}\left(-\operatorname{dom} g^{*}\right) \neq \emptyset
$$

imply

$$
L \cap \operatorname{cl}(\operatorname{Im} \partial h) \neq \emptyset ?
$$

Question $3 .^{2}$ Does the nonsmooth squeeze result of Corollary 4.1 hold more generally for any $n, k$ ?

\section{REFERENCES}

[1] J. M. Borwein And S. P. FitzPatrick, Duality Inequalities and Sandwiched Functions, preprint, 1999.

[2] F. H. Clarke, Optimization and Nonsmooth Analysis, John Wiley and Sons, New York, 1983.

[3] F. H. Clarke and Yu. S. Ledyaev, Mean value inequalities, Proc. Amer. Math. Soc., 122 (1994), pp. 1075-1083.

[4] J. B. Hiriart-Urruty and C. Lemaréchal, Convex Analysis and Minimization Algorithms I, Grundlehren Math. Wiss., 305, Springer-Verlag, Berlin, 1993.

[5] J. B. Hiriart-Urruty and C. Lemaréchal, Convex Analysis and Minimization Algorithms II, Grundlehren Math. Wiss., 306, Springer-Verlag, Berlin, 1993.

[6] A. S. Lewis AND D. RAlPh, A nonlinear duality result equivalent to the Clarke-Ldyaev mean value inequality, Nonlinear Anal., 26 (1996), pp. 343-350.

[7] R. T. Rockafellar, Convex Analysis, Princeton University Press, Princeton, NJ, 1970.

\footnotetext{
${ }^{2}$ After this paper was submitted, this question was resolved in the affirmative in [1].
} 